\title{
The Ordinal Interpretation of the Integers and Its Use in Number Theory
}

\author{
Nathan Thomas Hamlin \\ Department of Mathematics, Washington State University, Pullman, WA, USA \\ Email: n.hamlin@wsu.edu
}

How to cite this paper: Hamlin, N.T. (2019) The Ordinal Interpretation of the Integers and Its Use in Number Theory. Open Journal of Discrete Mathematics, 9, 165-175.

https://doi.org/10.4236/ojdm.2019.94013

Received: June 3, 2019

Accepted: October 25, 2019

Published: October 28, 2019

Copyright $\odot 2019$ by author(s) and Scientific Research Publishing Inc. This work is licensed under the Creative Commons Attribution International License (CC BY 4.0).

http://creativecommons.org/licenses/by/4.0/

\begin{abstract}
The author recently published a paper which claimed that an ordinal interpretation of numbers had limited applicability for cryptography. A further examination of this subject, in particular to what extent an ordinal interpretation is useful for recurrence sequences, is needed. Hilbert favored an interpretation of the natural numbers that placed their ordinal properties prior to their cardinal properties [1] [2]. The author examines ordinal uses of the integers in number theory in order to discuss the possibilities and limitations of this approach. The author hopes this paper will be useful in clarifying or even correcting some matters that were discussed in his paper of January of 2018. I was trained informally in philosophical realism, and while I think idealism too has a place, at this time in my life I believe that the weight of evidence and usefulness is more on the side of philosophical materialism. I hope this discussion will help supplement for my readers the material in Number in Mathematical Cryptography. I still maintain that a lack of clarity on these matters has hindered progress in cryptography; and it has taken time for me to better understand these things. I hope others who have interest and ability will assist in making these matters clearer. My intention was to work in pure mathematics, and the transition to an applied mindset was difficult for me. As a result, I feel most comfortable in a more middle-of-the road attitude, but have had to slowly move to a more precise analysis of the physical quantities involved. I hope my readers will be patient with my terminology, which is still evolving, and my discussion of things which are more indirectly related, and which are necessary for my expression. These are important things for the mathematical community to understand, and I hope smarter and more knowledgeable people will address my errors, and improve upon the things I might have correct. I am discussing sequences which are sometimes a use of both ordinal and cardinal numbers.
\end{abstract}

\section{Keywords}

Ordinal Interpretation, Integers, Number Theory, Hilbert's Theory 


\section{Introduction}

\subsection{Transcendence in Music}

I am going to begin this discussion with music, because it is more accessible than the technical use of number, but also in order to draw on the work of talented early 20th century thinker and writer, Adolf Reinach. This analysis will not be strictly phenomenological, but I will use that as a starting place, in part because it is our first experience of the entities involved. So, suppose you are hearing the note $\mathrm{C}$ played on a violin. We are not concerned about in which octave, but in some particular octave. Physically speaking, the sound enters your ear. Psychologically speaking, you experience a long, sweet, sound. If you have perfect pitch, perhaps you can even identify that tone as " $\mathrm{C}$ " without any further information. For most of us, we might have to look at where the violinist has her finger or make use of a device which measures the frequency of the sound, and perhaps converts that number into a letter on the scale. Is the note " $\mathrm{C}$ " a sweet sound that we hear, or is it a wave of a particular frequency traveling through the medium of air? The scientist is more satisfied with the latter, the music lover perhaps the former, though when pushed to it the music lover might concede that the scientific description is the correct one, objectively speaking. We can add that the experiences are in the brain or nervous system, but they are still distinct from measurements of frequency made outside the body. This analysis can be extended to chords such as $\mathrm{C}$ major, and Reinach; student of Husserl does just that [3] in a way that brings unusual clarity to the experience of listening to music. In terms of transcendence, there is a physical wave somehow behind the experience of that sweet sound, and people were able to enjoy music before we had much understanding of wavelength and frequency. The musical score before the violinist, of notes in positions on the scale, and relative temporal length, gives direct information for her hands to find the positions on her instrument. If she plays well, the air will be disrupted in a particular way and her audience will hear beautiful music. The dots on the page called musical notes refer to something off the page: finger positions on a wooden instrument, frequencies of waves moving through air, and finally a qualitative experience for the listener. This latter is an object of knowledge. If I am familiar with the piece from Mendelson, I may be able to evaluate whether the violinist played it properly. This perhaps over-involved description allows us to grasp the relationship between musical notation, a particular state an instrument is in, physical properties of waves, and finally, characteristic psychological experience.

\subsection{Number as an Ideal Entity}

The situation is not exactly the same with number, but similar; and there is some circularity because our understanding of tone goes back to the Pythagoreans, who discovered the string ratios for some of the most basic musical arrangements. However, our modern use of number in machines makes this an arguably helpful metaphor. The earliest computers were analog, based on a continuum, 
but the digital computer became more important. We want to look at how it is that earlier number theory, which as late as the 1930s was being declared useless (really, militarily speaking) easily becomes very important for a digital machine. We could examine the psychological experience of number, Reinach's teacher's concern, but it is actually not essential to what we have to say here, although I would invite readers who are number theorist or involved in combinatorics to consider whether there is some similarity to our experience of a particular natural number to that of a musical tone. Here, I am trying to move easily from mathematical notation perhaps in a textbook to machine states and possibilities. I'm being somewhat vague here, but in context it should be fine.

An ideal entity is something found in immediate human experience but which transcends that experience in a way we may or may not understand.

I am more comfortable with the practical usefulness of this definition than its theoretical value.

Here I am claiming that number can be an ideal entity. It's actually not necessary for the purposes of computing for us to consider numbers we can easily bring before our mind. Suppose you have some numbers written on a page, and one of them is $2^{101}$. That's easy notation for a number theorist, and we might even start pondering some of the properties of that number, or related numbers. But if this refers to the number that our handheld computer currently has in memory (say, for example, someone working on that computer just wrote down the number in memory on a piece of paper and left it on the desk) then it has a concrete physical meaning that is related but distinct from the writing on the page. The symbols on the page become part of our experience, they refer to a state in hardware which we may or may not understand: a switch that is on followed by one hundred that are off.

Notice that an ideal entity could be something real. The object or subject may be removed in time or space from the experience. The author has tried to avoid a metaphysical discussion of number, mostly because of the professional consensus that such concerns belong to Philosophy. But in our time philosophers are much more concerned about physics than mathematics, and mathematics is not a subfield of physics. It's been around much longer for example and has other concerns. As someone who has held both Platonic and Epicurean views, and currently favors the latter for understanding most sciences, let me at least point the way to the discussion that I believe needs to take place. Plato and Pythagoras advanced a theory of number that made it logically or metaphysically prior to matter. Hardy's view of number theory as a field of knowledge with no application is not very far from this, though admittedly he is not in line with much modern thought about number. The view can be held with or without a mystical attitude towards numbers. If numbers are merely constructed, why do they confront us in our experience of both physical and biological reality at both the micro and macro level? Why are the simple geometric exercises left to us by the Greeks so compelling? If they are mere abstractions, why are operations with them so much more reliable than operations with other universals? That is, why 
do number theory and analysis not fall prey to the same problems that plagued scholasticism? Precision doesn't seem to be the only issue. Why is the methodology primarily not experimental? A realist account can answer these questions, perhaps not to the satisfaction of all parties concerned. But some answer is needed, and something more than changing the subject is required. Maybe our study of physics will eventually explain our experience of number. But why would it, and does it seem to be moving in that direction? Why is Plato's view still sufficient for mathematics instruction for the most part, whereas modern views are mostly useful in specific situations: e.g. precision about the nature of the square root of 2 ? The author is mostly avoiding these questions in favor of examining the relation of number to computing. Perhaps they can be ignored altogether by most practicing mathematicians. But when these issues were last settled, in Hardy and Hilbert's time, computing was not part of the discussion, and analysis could in some sense be identified with the applicable part of mathematics. That is, the question "how does analysis relate to physics and engineering?" was the compelling question that was also thought to encompass the nature of mathematics. It was answered by mathematicians back then; perhaps similar questions can, and should, be answered by mathematicians today. At the very least, computing brings the natural numbers back to the center of mathematics. Theorems from Hardy's book, which he claimed would never have an application, were used in the development of the RSA cryptosystem. Why is that so? And can it be so, really, if Hilbert and Hardy were completely correct in their understanding of the metaphysics of number? Maybe the metaphysical questions can only be avoided for a time, in the context of the actual advancement of the sciences. The author is not a formally trained philosopher, and admits that he may not know what the status of these questions is in philosophy of mathematics. My concerns are admittedly directed toward a proper appreciation of number theory within the fields of mathematics and computer science.

\subsection{Ideality in Quantum Computing}

Computing became easier with the identification, by convention, of hardware states with numbers. In conventional computing, you have small switches where the "on" state is identified with " 1 " and the "off" state is identified with " 0 ": different switches representing different powers of 2 . In quantum computing, a third possibility " 0 or 1" probably transcends most peoples' experience as it refers to something on the molecular level that is comprehensible only within advanced physics. The author believes it is an open question whether we really have the best notation for this yet. But since Microsoft has released a quantum computing programming language, perhaps it is sufficient notation. It can be confusing in discussing because it does not refer to an "on or off" situation of a switch, and so it not analogous to the use of 0 and 1 in conventional computing.

\subsection{Ordinality and Temporality}

It might be worthwhile to include a brief discussion of time in this context: 
musical notes and chords being successively played in time, machine states following one after another in time, the ordinal numbers being "counted off" in time. Here I will be a little esoteric for the sake of the actual historical discussion. Euler held arguably rather Platonic views on some things, and had a good understanding of analysis, so we are not necessarily moving back to Medieval mathematics if we examine the views that were frequently held prior to the late 19th and early 20th century. There is much that could be said here and I am just going to be brief, at the expense perhaps of being as clear as I would prefer. It's worth noting that some people who have made significant contributions to our understanding of number have held rather esoteric or even bizarre views on the nature of both the temporal and the continuum: e.g. Pythagoras, Plato, Augustine, perhaps Pascal. Even Euclid can be difficult for a student of modern mathematics to follow. The limiting process, which proved so useful in understanding the continuum, was introduced to solve problems that were already intuitively understood in some cases. Earlier thinkers examined time in a context in which devices to measure it had more extensive limitations than today. The real line is perhaps most easily associated with time in the process of math and science education. In terms of the natural numbers, the view that ordinality is a temporal order is more plausible than the suggestion that it is a spatial order. In human experience, ordinality is largely connected to the temporal order, and perhaps this is what constrains us to project ordinality on the natural numbers. We can only do a limited amount of thought in a limited amount of time, and thus cannot grasp an infinite sequence except in parts which approach the whole. Unless some otherworldly form of consciousness exists such as Plato had envisioned, we probably should be willing to ascribe this to reality as a whole, and not merely to human experience: i.e. to assume an ordinal property for each natural number and for each real number, that goes beyond mere relation (<etc. which has to do with a comparison of cardinality). Thus, ordinality may not be an intrinsic property of the numbers, but we might have every reason to think that it is. On the other hand, cardinality may be more adequate to immediate experience, but not entirely adequate for explaining the use of number in most contexts we are actually concerned about. This is somewhat reversing my firmer earlier stance that cardinality was necessarily more fundamental than ordinality. Maybe it's just improving upon it. With numbers, as with music, one can be carried by the beauty into views which account for experience fairly well but do not necessarily hold up as solidly as material considerations when looking for a theory to explain the whole. In terms of the natural numbers as we have them in computing, they are counted by a process in time of successive machine states, related to one another by a very simple algorithm. The same of course is true of them as we know them in counting verbally, on our hands, or on paper. That we are often seeking to arrive at a particular cardinal number may only demonstrate that cardinality is usually more fundamental in our concerns. In terms of cryptography, the physical testing over time transcends the algorithms and formulas which we have on paper. 
These provide and important and essential source of evidence for the security of a code. A distinction in the field of cryptography is that we don't necessarily know who does or does not have that evidence available to them, at least until it has been released publicly.

\subsection{Historical Context for Hilbert's Theory: Materialism for the Hard Sciences}

The two well-defended metaphysics, with associated epistemologies, that came out of the 19th century were materialism and idealism. Idealism was, and is, believed to be extremely viable for the arts [4]; materialism was and is favored for the hard sciences. The connection of mathematics with the hard sciences made materialism an attractive working philosophy for mathematics, even though mathematical entities are arguably ideal.

Official epistemologies are arguably not in general essential on either a pedagogical or practical level in applied mathematics, which may be why epistemological and metaphysical concerns are often viewed as irrelevant in a professional mathematical context.

The author believes that an idealist approach to number can be safely dismissed in the Academy for the purposes of number theory.

\section{Number Theory and Computer Science}

Prior to digital computers and public-key cryptography, number theory was thought to be in the "extremely pure" category of mathematics [5], if not a form of recreational mathematics. Its contemporary applications are thus, from an important perspective, paradoxical under the assumptions of the early 20th century. In some ways even our legislation has not caught up with the new situation (if it needs to catch up).

Looking at Figure 1, there is a meaningful contact with the physical matter to be restructured in steps (4) and (5), and possibly in 3; but it appears that (2) is an ideal process, except in so far as it is recorded on paper or word processing software, which seems incidental, especially for very simple (as opposed to compound) results. In such a case, a materialist account of mathematical entities might obscure the most important steps in the design and implementation process. A careful, limited, realism might be a useful alternative.

The field as a whole (1) does not exactly exist on paper, and it is regarded in practice as ideal: someone generally gets the credit, but not the ownership of a theorem, and this is in some ways more analogous to the situation in the arts than in the hard sciences, such as physics, engineering, and chemistry. Does "pure" mathematics take us farther from the sciences, but closer to the arts?

Incidentally, (6) is a social process that intersects with both politics and economics, and those who represent such interests in the university and perhaps other forms of leadership.

A poem is an ideal entity, and may have economic value. When purchasing an 


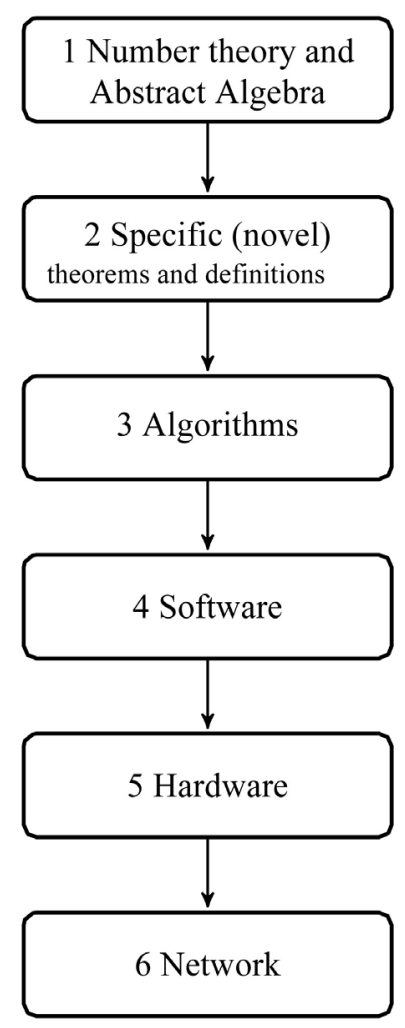

Figure 1. Structure of a cryptographic system.

anthology of Byron, for example, the author at least would not be primarily interested in the value of the paper. Byron seems to be in the public domain, and probably for some arguably good reasons. Do similarly good reasons place the applicable mathematical content of a Ph.D. thesis in the public domain during the author's working years? Can an author or publisher own a poem? These issues become challenging with respect to the presence of the internet in particular. More, generally, if other things of economic value, sold commercially, are going to be produced using the poem or theorem-say in the technical arts or in a computer science application-there are questions of ownership that the author believes are analogous in some ways to citations in academic work. Our current patent laws, to the author's admittedly limited knowledge, mostly extend to the level of (4) or perhaps (3).

\section{Limitations of the Realist Approach}

The main problem with a realist theory of numbers is the one that is well-known: it generally brings us into the transcendental at the very moment we need to be in the material. This can be avoided, and the author believes he has avoided it [6]. But it was a more serious concern earlier in the 20th century, and this may also have been a motivation for Hilbert. At the time, number theory was thought to have very limited application [5], and there was really no good reason to think that a realist interpretation of the integers would increase the viability of the field. Husserl's concern [7] about "grounding" the sciences proved not to be as 
pressing of an issue as he believed, though one might come to similar conclusions as Husserl in the need to coordinate conceptions of number in different fields, as was the case for the author. In the author's understanding Husserl and his realism is not embraced by most mainstream philosophers at this time. So long as this move is not perceived as an invitation to second-rate philosophers into the applied sciences or their supporting philosophy, the author believes this will not be a problem.

\section{Sequences}

Let us take for our example the combinatorial Fibonacci numbers [8]:

$$
\begin{gathered}
f_{n}=f_{n-2}+f_{n-1} ; f_{1}=1, f_{2}=2 \\
\left\{f_{n}\right\}=\{1,2,3,5,8,13,21,34,55,89, \cdots\} \\
\left\{f_{n}\right\}=f_{1}, f_{2}, f_{3}, f_{4}, f_{5}, f_{6}, f_{7}, f_{8}, f_{9}, \cdots
\end{gathered}
$$

In Equation (3) we have the positive integers being used as ordinal numbers. Some of this borders on the trivial, but it is putting it all together properly that is important. The subscripts do not merely distinguish distinct Fibonacci numbers from one another, but indicate a relationship between the Fibonacci numbers. The combinatorial Fibonacci numbers are in fact an increasing sequence, so that relationship corresponds to the relation <, but this is merely incidental since there are other sequences, such as the powers of $1 / 2$ or of -2 , for which that would not be the case. So, this order cannot be reduced to an ordering, and apart from the possibility of functional notation it doesn't seem like there is any mathematical reason to prefer the natural numbers to another suitable subset of the real numbers. Their usefulness and applications are more universal than many other subsets of the real numbers.

We may consider this in terms a little more general:

$$
\left\{u_{n}\right\}=u_{1}, u_{2}, u_{3}, u_{4}, u_{5}, u_{6}, u_{7}, u_{8}, u_{9}, \cdots
$$

where $u_{n}=u_{n-4}+u_{n-3}+u_{n-2}+u_{n-1}$ or $u_{n}=u_{n-16}+u_{n-12}+u_{n-10}+u_{n-1}$ with appropriate initial conditions.

Essentially, (3.3) makes explicit some important relationships between members of (3.2) that depend on the more fundamental relationship expressed in

The author recognizes that there are probably many things that Hilbert understood better than him, but he would like to point out that the 4th Fibonacci number is not only, or even essentially, characterized by being the Fibonacci number that comes after the third, or before the fifth. For one thing, it has an important fundamental relationship with both the second, and the sixth (trivial). More generally, the fourth Fibonacci number has many important relationships with every other member of the sequence.

In other words, the natural numbers, for some of the most important purpose we use them as ordinal numbers, cannot be reduced to mere succession [1] [2]. So even if the cardinal numbers could be reduced to ordinal numbers [1] [2], 
that would actually raise more questions than it answers, and the author has given ample reason elsewhere [6] why this cannot be done because of important mathematical and technical concerns.

\section{Integers as Quantities}

In dealing with particular integers, we encounter a seemingly more atomistic situation, though if we are to allow that the same integers which refer to quantities can enter into relations in sequences as described in the previous section, then we must prefer an individualist over an atomistic account. It will be helpful to examine a situation highly relevant to cryptography, in which both uses of numbers, the ordinal and cardinal, are required in the same problem.

The method of cryptography I have somewhat informally referred to as the Generalized Knapsack Code has been introduced elsewhere [9]. It is based on the possibility of representing integers using more or less arbitrary (linear) recurrence sequences.

\section{An Example Representation}

Let $\left\{u_{i}\right\}$ satisfy the recurrence $u_{n}=u_{n-1}+3 u_{n-3}+2 u_{n-4}$ (the initial values are not vital, but the standard ones are generally more beautiful and convenient, i.e., $\left.u_{0}=u_{1}=u_{2}=1\right)$. The signature is $S=1032$. The representation of any positive integer $M$ is of the form

$$
M=\sum_{i=0}^{n-1} d_{i} u_{i}
$$

where the string of digits $d_{n-1} d_{n-2} \cdots d_{1} d_{0}$ must be composed of blocks of digits which are lexicographically smaller than $S$. In this case, the allowed blocks of digits are $0,100,101,102,1030,1031$. Hence, for instance, 102|1030|0|101 is a legitimate string, but 101|1102103 is not. Notice that no allowed block begins with 11.

This context will allow us to examine how ordinal and cardinal numbers work together in a context that is arguably of some importance for elementary number theory, analytic number theory, and cryptography [9].

Here the ordinal structure of the $u_{k}$ indicates to the reader, cryptographer, or cryptanalyst what significance should be assigned to the $d_{i}$ from the underlying recurrence sequence.

More generally with respect to place value, say for base 10, the sequence of places produces a context of interpretation for each digit, which cannot be reduced to the relationship that each digit has with its adjacent digits. So for example, when considering the integer represented in base 10 by 34,782 , the numeral 7's interpretation as 7 hundreds cannot be reduced to its proximity to 4 and 8 , though we can determine from experience and theory that the hundreds place is between the tens' place and the thousands' place.

\section{Conclusions}

These questions and the possible answers to them are often thought to properly 
belong to philosophy. But there are times when a mathematician needs an account of number, and his or her needs, or at least intentions, are likely to differ from those of the philosopher. Public-key cryptography may pose a fundamentally different challenge for mathematics and mathematicians than we have previously faced, and it may be necessary to resolve some issues "in house" that were once left to others. Whether or not this appeals to analysts, it is a matter of importance for elementary number theory and its applications, which are immense. The issues can be resolved for our purposes, and perhaps those of computer scientists, without an appeal to metaphysics, or challenging the notion that the primary applications of the sciences are material. However, in the context of "virtual" communication and "virtual" transactions which rely upon number theory at the most abstract level, a certain amount of clarity is required for issues that once might have been regarded as too soft for this kind of analysis.

The author believes that the proper point to be taken from Hardy's excellent discussion [5] is that number theory is a creative field, whose value cannot be reduced to its applicability, whether economic or political. Hardy, and also the excellent Kinchin (Three Pearls of Number Theory), express a realist perspective of numbers in practice. The author believes there may be something of divide in the field between number theorists and analysts on this matter, which might explain the divergence of Hilbert's views. A number theorist might view the real line as a different kind of object, a continuum for example, which could account for the need of a different kind of discussion of ordinality and cardinality. The author hopes that some mathematicians will agree that most of these concerns belong to mathematics properly, and find the non-mathematical analogies useful.

\section{Conflicts of Interest}

The author declares no conflicts of interest regarding the publication of this paper.

\section{References}

[1] Ewald, W. (1996) From Kant to Hilbert: A Source Book in the Foundations of Mathematics. Vol. II, Clarendon Press, Oxford.

[2] Husserl, E. (2003) Philosophy of Arithmetic. D. Willard Translation, Kluwer Academic Publishers, Dordrecht.

[3] Reinach, A. (1969) Concerning Phenomenology. The Personalist, Vol. 50, 194-221.

[4] Derrida, J. and Attridge, D. (1992) Acts of Literature. Routledge, New York.

[5] Hardy, G.H. and Snow, C.P. (1967) A Mathematician's Apology. Cambridge University Press, London.

[6] Hamlin, N. (2017) Number in Mathematical Cryptography. Open Journal of Discrete Mathematics, 7, 13-31. https://doi.org/10.4236/ojdm.2017.71003

[7] Husserl, E. and Carr, D. (1970) The Crisis of European Sciences and Transcendental Phenomenology. Northwestern University Press, Evanston.

[8] DeTemple, D. and Webb, W. (2014 ) Combinatorial Reasoning: An Introduction to 
the Art of Counting. Wiley, Hoboken, NJ.

[9] Hamlin, N. (2016) Recurrence Representations: An Exploration of Number, Representation, and Public-Key Cryptography. Lambert Academic Publishing, Saarbrücken, Germany. 